\title{
Genetic predisposition scores for dyslipidaemia influence plasma lipid concentrations at baseline, but not the changes after controlled intake of n-3 polyunsaturated fatty acids
}

\author{
Aseel AISaleh · Zoitsa Maniou • Fiona J. Lewis • \\ Wendy L. Hall • Thomas A. B. Sanders • \\ Sandra D. O'Dell
}

Received: 19 February 2014/ Accepted: 22 May 2014/Published online: 3 June 2014

(c) Springer-Verlag Berlin Heidelberg 2014

\begin{abstract}
Inconsistent effects of fish oil supplementation on plasma lipids may be influenced by genetic variation. We investigated 12 single nucleotide polymorphisms (SNPs) associated with dyslipidaemia in genome-wide association studies, in 310 participants randomised to treatment with placebo or $0.45,0.9$ and $1.8 \mathrm{~g} /$ day eicosapentaenoic acid (20:5n-3, EPA) and docosahexaenoic acid (22:6n-3, DHA) (1.51:1) in a 12-month parallel controlled trial. Effects of risk alleles were assessed as trait-specific genetic predisposition scores (GPS) and singly. GPS were positively associated with baseline concentrations of plasma total cholesterol, low-density-lipoprotein cholesterol and triglyceride (TG) and negatively with high-density-lipoprotein cholesterol. The TG-GPS was associated with $0.210 \mathrm{mmol} / \mathrm{L}$ higher TG per risk allele $(P<0.0001)$, but no effects of single TG SNPs were significant at baseline. After treatment with EPA and DHA, TG-GPS was associated with $0.023 \mathrm{mmol} / \mathrm{L}$ lower TG per risk allele $(P=0.72)$. No interactions between GPS and treatment were significant; however, FADS1 SNP rs174546 $C / T$ interaction with treatment was a significant determinant of plasma TG concentration $(P=0.047, n=267)$. Concentration differed between genotype groups after the $1.8 \mathrm{~g} /$ day dose $(P=0.026)$, decreasing by $3.5(95 \% \mathrm{CI}-15.1$
\end{abstract}

On behalf of the MARINA study team.

Electronic supplementary material The online version of this article (doi:10.1007/s12263-014-0412-8) contains supplementary material, which is available to authorized users.

A. AlSaleh · Z. Maniou · F. J. Lewis · W. L. Hall ·

T. A. B. Sanders · S. D. O'Dell ( $\varangle)$

Diabetes and Nutritional Sciences Division, School of Medicine,

King's College London, Franklin-Wilkins Building,

150 Stamford Street, London SE1 9NH, UK

e-mail: sandra.odell@kcl.ac.uk; sandra.o'dell@kcl.ac.uk to 8.2$) \%$ in non-carriers of the risk $T$-allele $(n=30)$ and by $21.6(95 \% \mathrm{CI}-32.1$ to -11.2$) \%$ in carriers $(n=37)$, who showed a highly significant difference between treatments $(P=0.007)$. Carriers of the FADS1 rs174546 risk allele could benefit from a high intake of EPA and DHA in normalising plasma TG.

Keywords Docosahexaenoic acid - Eicosapentaenoic acid - Genetic predisposition score $\cdot$ Gene-nutrient interaction · Plasma lipids · Single nucleotide polymorphism

\section{Introduction}

Plasma lipids are risk factors for cardiovascular disease (CVD) and are well known to be influenced by dietary intake (Marais 2013). A large number of human intervention trials have shown that eicosapentaenoic acid (20:5n-3, EPA) and docosahexaenoic acid (22:6n-3, DHA) in fish oil exert cardioprotective effects (reviewed by Mozaffarian and Wu 2011; Delgado-Lista et al. 2012). However, some studies and meta-analyses have failed to demonstrate any significant protection from CVD risk by n-3 PUFA treatment (Kromhout et al. 2010; ORIGIN Trial Investigators et al. 2012; Rizos et al. 2012). Effects of fish oil on plasma lipids are also inconsistent (see Minihane (2013) for recent review) and this may reflect genetic variation as well as different treatments. Heritability estimates for fasting plasma lipids range from $32 \%$ for triglyceride (TG) to $55 \%$ for total cholesterol (TC) (Pérusse et al. 1997). Interaction between genetic and environmental factors such as dietary intake may contribute to the variation in lipid traits between individuals (Corella and Ordovas 2005). 
Genome-wide association studies (GWAS) have identified a number of single nucleotide polymorphisms (SNPs) associated with dyslipidaemia in cross-sectional studies (Chasman et al. 2008; Kathiresan et al. 2008, 2009; Wallace et al. 2008; Willer et al. 2008; Aulchenko et al. 2009; Sabatti et al. 2009; Teslovich et al. 2010). However, few attempts have been made to discover whether these common SNPs also influence the responses to dietary interventions. As intervention studies are necessarily limited in size, significant interactions with single SNPs may be difficult to establish, but interaction with sets of SNPs potentially offer more power. We hypothesised that carriage of alleles predisposing to dyslipidaemia may influence changes in the plasma lipid profile in response to intake of EPA and DHA. We tested the hypothesis in a cohort of 310 participants from the MARINA (Modulation of Atherosclerosis Risk by Increasing doses of N-3 fatty Acids) study (Sanders et al. 2011), a highly controlled randomised dietary intervention trial to investigate the effect of olive oil placebo and EPA and DHA (1.51:1) supplemented at $0.45,0.9$ and $1.8 \mathrm{~g} /$ day for 12 months on CVD risk factors in healthy subjects. We investigated effects of 12 SNPs with reported associations with plasma lipids at genome-wide levels of significance, in combination and individually, on plasma lipid concentrations at baseline and on changes in response to treatment.

\section{Methods}

Subjects

The MARINA trial was a single-centre dietary intervention study of randomised controlled double-blind parallel design, to test the effects of three daily doses of EPA and DHA for 12 months on endothelial function and CVD risk factors (Sanders et al. 2011). The study was conducted at King's College London between April 2008 and October 2010 and approved by the St Thomas' Hospital NHS Research Ethics Committee (NREC 08/H0802/3). Written informed consent was given by participants, who were healthy non-smoking men and women aged between 45 and 70 years, recruited and screened as described previously (Sanders et al. 2011). Subjects with untreated high blood pressure or raised cholesterol were excluded. 367 participants were randomised to treatment. During an initial run-in period of 4 weeks, participants took olive oil (British Pharmacopoeia specification) placebo capsules whilst restricting oily fish intake, after which baseline measurements of outcome variables were made. The proportion of energy derived from total fatty acids, saturated fatty acids and PUFA ascertained by food frequency questionnaire at baseline was similar to that of the general population, as reported previously (Sanders et al. 2011). During dietary intervention, capsules containing placebo or EPA and DHA (1.51:1) at three doses $(0.45,0.9$ and $1.8 \mathrm{~g} /$ day) were supplied at regular intervals. The present investigation was based on measurements made at baseline and after 12 months, and compliance was verified by assessment of EPA and DHA in erythrocyte phosphoglycerides at this time. Supply of oil blends and quality control analysis was by Croda Chemicals Europe Ltd. (Hull, UK) and encapsulation in gelatine was by Powerhealth (Pocklington, UK).

Measurement of serum lipoproteins and triglycerides

TC, high-density-lipoprotein cholesterol (HDL-C) and TG were analysed by enzymatic assay using methods described previously (Jebb et al. 2010). Inter-assay coefficients of variation for TC were 1.1, 1.5 and $1.0 \%$ at 3.9, 5.1 and $5.7 \mathrm{mmol} / \mathrm{L}$ respectively; for HDL-C, they were $2.2,2.1$ and $2.5 \%$ at concentrations of $0.91,1.39$ and $1.95 \mathrm{mmol} /$ $\mathrm{L}$, respectively; and for $\mathrm{TG}$, they were 2.5 and $1.5 \%$ at concentrations of 1.32 and $2.36 \mathrm{mmol} / \mathrm{L}$, respectively. Low-density-lipoprotein cholesterol (LDL-C) was derived from the Friedwald equation if fasting plasma TG concentrations were $<4.49 \mathrm{mmol} / \mathrm{L}$.

SNP selection and genotyping

Twelve lipid-associated SNPs were selected from six GWAS published prior to November 2012 (Kathiresan et al. 2008, 2009; Willer et al. 2008; Aulchenko et al. 2009; Sabatti et al. 2009; Teslovich et al. 2010). SNPs selected for genotyping had a minimum minor allele frequency (MAF) of 0.30 in subjects of European ancestry. At a given gene locus, the SNP with the strongest effect on the lipid trait was selected, taking into account both the sample size and the $P$ value, which in all cases was lower than the threshold for genome-wide significance $\left(P<5 \times 10^{-8}\right)$. Nine SNPs had reported associations with TC, 6 SNPs with HDL-C, 7 SNPs with LDL-C and 8 SNPs with TG. Some SNPs were associated with more than one lipid trait (see Online Resource 1). The 12 SNPs genotyped were either those reported in the GWAS, or proxies in strong linkage disequilibrium (LD) $\left(r^{2}>0.8\right.$ based on HapMap Phase II samples of European ancestry (International HapMap Project 2012)).

DNA was extracted from buffy coats as described previously (Al-Hilal et al. 2013). SNPs were genotyped in 310 participants for whom DNA was available. Genotyping was performed by KBiosciences (Hoddesdon, Herts, UK) using KASPar technology. Genotype accuracy, as assessed by inclusion of duplicates in the array, was $98 \%$, and negative controls (water blanks) were included on each 
plate. The genotyping success rate was $87-92 \%$. All genotype distributions were tested for deviation from the Hardy-Weinberg equilibrium.

\section{Genetic predisposition score (GPS)}

A risk allele was defined as the allele associated with higher plasma concentration of TC, LDL-C, or TG or lower concentration of HDL-C in previous GWAS (Kathiresan et al. 2008, 2009; Willer et al. 2008; Aulchenko et al. 2009; Sabatti et al. 2009; Teslovich et al. 2010) (shown in Online Resource 1). We elected not to weight the risk alleles on the basis of their individual effect sizes, as this may have only limited effect (Janssens et al. 2007). Also, the effect size of SNPs on traits at baseline may not be relevant to their effect on any change following treatment. A simple addition of the associated risk alleles for each trait carried by each individual, as described by $\mathrm{Li}$ et al. (2010), was used to calculate a GPS for each trait TC, HDL-C, LDL-C and TG. Individual SNPs were coded as 0,1 and 2 according to the number of risk alleles for that particular SNP. Fifty-eight out of 310 participants had missing data for more than one genotype out of 12 (more than $8.3 \%$ genotypes missing) and were excluded from the GPS analysis. For the 29 individuals missing one genotype among the remaining 252 participants, the average count of risk alleles for the respective SNP was substituted for the missing data in calculating the GPS, as adopted by Walker et al. $(2011,2013)$.

\section{Statistical analysis}

Genotype distributions were tested for Hardy-Weinberg equilibrium using a $\chi^{2}$ test with $1 d f(P>0.05)$ and did not deviate from expectations. Statistical analyses were carried out using the SPSS version 20.0 for Windows (SPSS Inc, Chicago, IL, USA). Distributions of outcome variables and trait-specific GPS were assessed for normality. Baseline TG was $\log (n)$-transformed for analyses and presented in tables in this form except for Table 1 Characteristics of study participants, where the geometric mean was shown. Associations between the GPS and respective traits at baseline and changes following treatment with EPA and DHA were tested by linear regression, adjusting for BMI, age, gender and ethnicity at baseline and additionally baseline values for change in variables after treatment. The three doses of EPA and DHA were compared to the olive oil placebo group, adding each treatment to the model individually. Linear regression analysis was also used to investigate effects of each SNP risk allele on the relevant trait at baseline and after treatment, assuming an additive effect of each additional risk allele and adjusting for the same covariates as in the GPS analysis. The proportion of variance in plasma lipid concentrations at baseline and in the change from baseline to 12 months explained by each GPS and by each individual SNP was estimated from the $r^{2}$ value of each regression model. We conducted the single SNP analysis recognising the limited power available to detect individual effects, in order to investigate their contribution to the effects of the GPS. We report these exploratory findings with no correction for multiple testing. Data presented in text and tables are expressed as mean $\pm \mathrm{SD}$ or geometric mean $\pm \mathrm{SE}$ and allele effects as beta coefficient with SE. Significance was taken as $P<0.05$.

\section{Results}

\section{Characteristics of subjects}

Table 1 shows the characteristics of subjects at baseline after a 4 week run-in on placebo. Data are presented for the 310 participants out of 367 randomised to treatment who completed the study and for whom DNA was available. The number of participants allocated and drop-out rates did not differ significantly between treatment groups (Sanders et al. 2011). Women, mostly postmenopausal, outnumbered men by approximately $1.6: 1$ and about $20 \%$ of the sample was non-white, with similar proportions of Asian and black participants. Based on self-reported ethnicity, we distinguished individuals of white (81.9\%), Far Eastern (5.2\%), South Asian (5.8\%); black (2.9\%) and other (4.2\%) ancestry. The average BMI was above the desirable range $\left(20-25 \mathrm{~kg} / \mathrm{m}^{2}\right)$, and the mean waist circumferences were greater than cut-offs indicating risk of metabolic syndrome (94 cm in men and $80 \mathrm{~cm}$ in women) (Sanders et al. 2011).

\section{SNP allele and genotype frequencies}

Twelve SNPs were genotyped. The minor allele and genotype frequencies in participants with DNA $(n=310)$ are shown in Online Resource 2. Genotype distributions did not deviate from Hardy-Weinberg expectations and minor allele frequencies were in close agreement with those listed for Europeans on the NCBI SNP database (2012).

Effect of genetic predisposition on plasma lipid concentrations at baseline

The distributions of trait-specific GPS and variations in plasma concentrations of TC, HDL-C, LDL-C and TG at baseline are shown in Fig. 1. All GPS were normally distributed. The higher the GPS, the less favourable the lipid profile. Effect sizes per risk allele at baseline are shown in 
Table 1 Characteristics of study participants at baseline by randomised treatment

\begin{tabular}{|c|c|c|c|c|c|}
\hline & $\begin{array}{l}\text { Placebo } \\
n=70\end{array}$ & $\begin{array}{l}0.45 \mathrm{~g} / \text { day } \\
n=80\end{array}$ & $\begin{array}{l}0.9 \text { g/day } \\
n=80\end{array}$ & $\begin{array}{l}1.8 \mathrm{~g} / \text { day } \\
n=80\end{array}$ & $P^{*}$ \\
\hline \multicolumn{6}{|l|}{ Gender } \\
\hline Male, $n(\%)$ & 31,44 & $30,37.5$ & $30,37.5$ & 29,36 & \\
\hline Female, $n(\%)$ & 39,56 & $50,62.5$ & $50,62.5$ & 51,64 & \\
\hline \multicolumn{6}{|l|}{ Ethnicity } \\
\hline White, $n(\%)$ & $57,81.4$ & $64,80.0$ & $64,80.0$ & $69,86.25$ & \\
\hline Far Eastern, $n(\%)$ & $6,8.6$ & $4,5.0$ & $5,6.25$ & $1,1.25$ & \\
\hline S. Asian, $n(\%)$ & $3,4.3$ & $6,7.5$ & $8,10.0$ & $1,1.25$ & \\
\hline Black, $n(\%)$ & $2,2.85$ & $3,3.75$ & $0,0.0$ & $4,5.0$ & \\
\hline Other, $n(\%)$ & $2,2.85$ & $3,3.75$ & $3,3.75$ & $5,6.25$ & \\
\hline Age (years) & $55.4 \pm 6.9$ & $55.1 \pm 6.8$ & $55.2 \pm 6.6$ & $55.1 \pm 6.7$ & 0.99 \\
\hline BMI $\left(\mathrm{kg} / \mathrm{m}^{2}\right)$ & $26.1 \pm 3.7$ & $25.1 \pm 3.9$ & $26.2 \pm 4.0$ & $25.2 \pm 3.5$ & 0.17 \\
\hline Waist circumference $(\mathrm{cm})$ & $89.5 \pm 11.7$ & $87.3 \pm 11.8$ & $89.4 \pm 11.9$ & $88.0 \pm 11.0$ & 0.60 \\
\hline \multicolumn{6}{|l|}{ Plasma lipids (mmol/L) } \\
\hline $\mathrm{TC}$ & $5.4 \pm 1.0$ & $5.4 \pm 1.1$ & $5.4 \pm 0.9$ & $5.4 \pm 1.0$ & 0.94 \\
\hline HDL-C & $1.6 \pm 0.5$ & $1.6 \pm 0.4$ & $1.7 \pm 0.5$ & $1.7 \pm 0.5$ & 0.26 \\
\hline LDL-C & $3.3 \pm 0.8$ & $3.2 \pm 0.8$ & $3.2 \pm 0.8$ & $3.2 \pm 0.7$ & 0.79 \\
\hline $\mathrm{TG}^{\mathrm{a}}$ & $1.2 \pm 0.5$ & $1.1 \pm 0.5$ & $1.1 \pm 0.4$ & $1.1 \pm 0.5$ & 0.96 \\
\hline
\end{tabular}

Data are presented for subjects randomised to treatment groups for whom DNA was available $(n=310)$. Measurements taken at baseline after 4-week run-in on normal diet with olive oil placebo supplement are shown for each randomised treatment group: placebo and EPA and DHA $(1.51: 1)$ at the daily doses shown. Values are $n(\%)$ for gender and ethnic groups, or mean \pm SD for all other variables except TG

$B M I$ body mass index, $H D L-C$ high-density-lipoprotein cholesterol, $L D L-C$ low-density-lipoprotein cholesterol, $T C$ total cholesterol, $T G$ triglyceride

* Significance of differences between treatment groups assessed by one-way ANOVA. $P$ value for difference in age, adjusted for BMI, gender and ethnicity; for differences in BMI and waist circumference, adjusted for age, gender and ethnicity; for differences in plasma lipid concentrations, adjusted for BMI, age, gender and ethnicity

${ }^{a}$ TG data not normally distributed and presented as the geometric mean $\pm \mathrm{SE}$

Table 2. The only significant effect of a GPS risk allele was on plasma TG concentration $(P<0.0001)$, so the remainder of the analysis focused on this trait. GPS risk allele carriage accounted for $20.5 \%$ of variance in TG concentration at baseline.

SNP associations with plasma lipid concentrations at baseline

None of the eight SNPs contributing to the TG-GPS showed a significant effect on plasma TG concentration at baseline (Online Resource 3). The effects of only two individual SNPs on their respective traits reached nominal significance: GCKR rs1260326 on TC and TRIBI rs2954029 on HDL-C.

Effect of genetic predisposition on the change in plasma lipid concentrations in response to treatment

Effect sizes per GPS risk allele after 12 months treatment with EPA and DHA are shown in Table 3. The effect was not significant for any trait, but deleterious effects of all risk alleles on plasma lipids were reduced after treatment. The hyperlipidemic effect of the risk allele on plasma TG concentration seen at baseline was reversed after treatment. Each additional risk allele was associated with $0.023 \mathrm{mmol} / \mathrm{L}$ lower TG after treatment $(P=0.72)$, in contrast to the higher concentration at baseline. GPS risk allele carriage accounted for $3.2 \%$ of variance in TG after treatment, a much lower proportion than at baseline. Interaction between GPS and treatment was not a significant determinant of plasma TG concentration $(P>0.05)$.

SNP associations with plasma lipid concentrations after treatment

We found no significant effects of single SNP risk alleles on plasma TG concentration (Online Resource 4). We then investigated interaction between treatment and individual TG SNP genotypes. Interaction between FADS1 rs 174546 $C / T$ and treatment was a significant determinant of plasma TG concentration $(P=0.047, n=267)$. The $T$-allele was associated with higher TG in the GWAS (Teslovich et al. 2010). There were no significant differences in the change 
(a) Effect $=0.11 \mathrm{mmol} / \mathrm{L}$ per allele, $P=0.07$

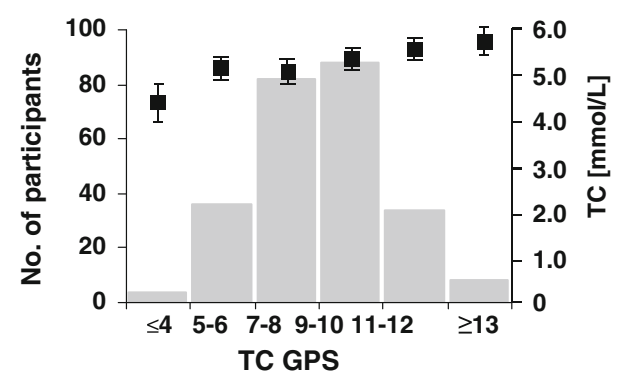

(c) Effect $=0.07 \mathrm{mmol} / \mathrm{L}$ per allele, $P=0.03$

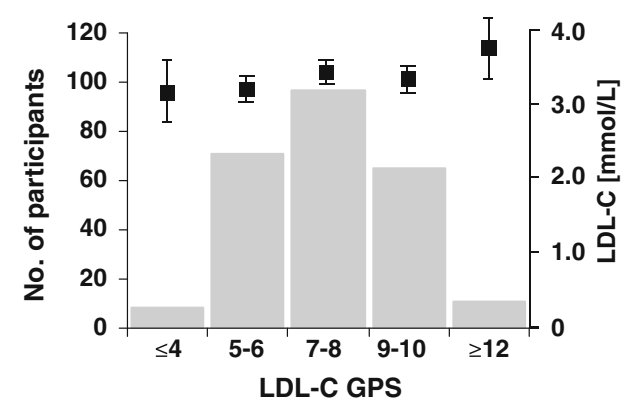

Fig. 1 Variation in plasma concentrations of a TC, b HDL-C, c LDL-C and $\mathbf{d}$ TG at baseline by trait-specific GPS. The number of participants for each GPS is indicated by the bars corresponding to the left-hand $Y$-axis. Data points are the mean and standard error (SE) values of plasma concentrations of a TC, b HDL-C, c LDL-C and d TG for each GPS score category defined by the number of risk alleles per individual, with units indicated on the right-hand $Y$-axis.

Table 2 Effect of GPS on plasma TC, HDL-C, LDL-C and TG concentrations at baseline

\begin{tabular}{lrlcll}
\hline & Effect & SE & $P^{*}$ & $r^{2}$ & $n$ \\
\hline TC $(\mathrm{mmol} / \mathrm{L})$ & 0.114 & 0.032 & 0.07 & 0.056 & 252 \\
HDL-C $(\mathrm{mmol} / \mathrm{L})$ & -0.011 & 0.015 & 0.08 & 0.306 & 252 \\
LDL-C (mmol/L) & 0.065 & 0.028 & 0.30 & 0.033 & 252 \\
lnTG $(\mathrm{mmol} / \mathrm{L})^{\mathrm{a}}$ & 0.210 & 0.013 & $<0.0001$ & 0.205 & 252 \\
\hline
\end{tabular}

Data show associations of GPS with each trait, presented as per allele effect size, and SE derived from linear regression models of GPS against trait at baseline. The proportion of explained variance is indicated by $r^{2}$ and $n$ is the number of participants in each subgroup. A negative effect represents an association with reduction in trait at baseline

$H D L-C$ high-density-lipoprotein cholesterol, $L D L-C$ low-densitylipoprotein cholesterol, $T C$ total cholesterol, $T G$ triglyceride

* Significance of per allele effect on change in trait derived from linear regression models, nominal at $P<0.05$. $P$ values adjusted for BMI, age, gender and ethnicity

${ }^{\text {a }}$ TG data were logged for the analysis and were presented as $\log (n)$ transformed data

from baseline between non-carriers and carriers of the risk allele after the placebo, 0.45 and $0.9 \mathrm{~g} /$ day EPA and DHA doses, but the change from baseline between non-carriers (b) Effect $=-0.11 \mathrm{mmol} / \mathrm{L}$ per allele, $P=0.08$

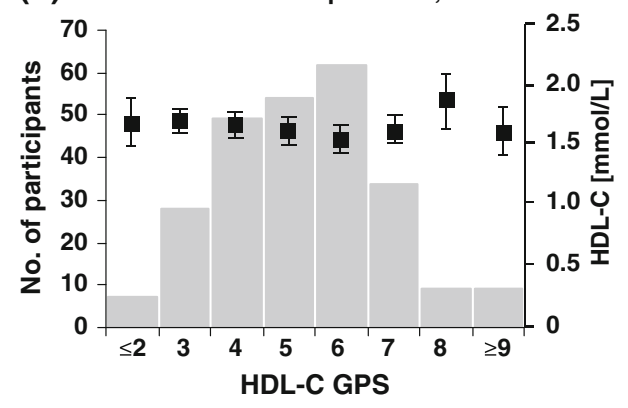

(d) Effect $=0.21 \mathrm{mmol} / \mathrm{L}$ per allele, $P<0.0001$

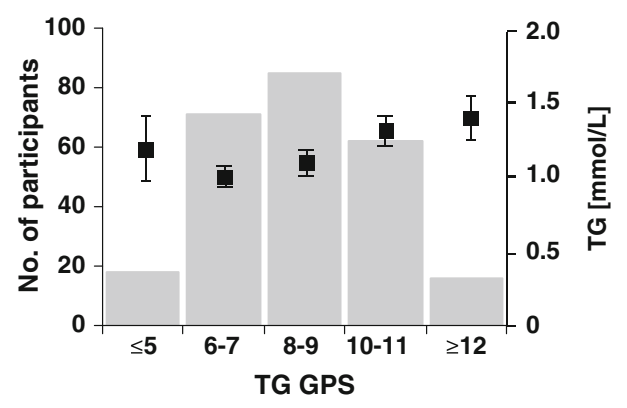

GPS at the lower and upper ends for each trait were grouped due to small $n$. The analysis was performed by linear regression of trait at baseline using the ungrouped GPS and adjusted for age, gender and ethnicity. The $P$ values for associations between plasma lipid concentrations and trait-specific GPS and effects per risk allele are indicated. $T C$ total cholesterol, $L D L-C$ low-density-lipoprotein cholesterol, $H D L-C$ high-density-lipoprotein cholesterol, $T G$ triglyceride

Table 3 Effect of GPS on change in plasma TC, HDL-C, LDL-C and TG concentrations after treatment

\begin{tabular}{lrllll}
\hline & Effect & SE & $P^{*}$ & $r^{2}$ & $n$ \\
\hline Change in TC (mmol/L) & 0.064 & 0.031 & 0.30 & 0.092 & 251 \\
Change in HDL-C (mmol/L) & 0.016 & 0.011 & 0.80 & 0.014 & 250 \\
Change in LDL-C (mmol/L) & 0.054 & 0.020 & 0.41 & 0.011 & 251 \\
Change in TG (mmol/L) & -0.023 & 0.011 & 0.72 & 0.032 & 251
\end{tabular}

Data show associations of GPS with change in each trait, presented as per allele effect size, and SE derived from linear regression models of GPS against change in trait. The proportion of explained variance is indicated by $r^{2}$, and $n$ is the number of participants in each subgroup. A negative effect represents an association with reduction in trait following 12-month intervention

$H D L-C$ high-density-lipoprotein cholesterol, $L D L-C$ low-densitylipoprotein cholesterol, $T C$ total cholesterol, $T G$ triglyceride

* Significance of per allele effect on change in trait derived from linear regression models, nominal at $P<0.05 . P$ values adjusted for trait value at baseline, BMI, age, gender and ethnicity

and carriers was significant after the $1.8 \mathrm{~g} /$ day dose $(P=0.026)$. In non-carriers $(n=30)$, plasma TG concentration decreased by $3.5 \%(95 \% \mathrm{CI}-15.1$ to 8.2$)$ from baseline, whereas in carriers $(n=37)$, TG concentration decreased by $21.6 \%$ (95\% CI -32.1 to -11.2$)$ and the 
difference between treatments was highly significant $(P=0.007)$.

\section{Discussion}

We investigated effects of 12 SNPs associated with plasma lipids at genome-wide levels of significance in 310 participants randomised to receive placebo or three doses of EPA and DHA for 12 months. A trait-specific GPS based on additive effects of risk alleles yielded a highly significant per allele effect for plasma TG concentration. EPA and DHA treatment had the greatest per allele effect on TG-GPS, reversing the direction of the effect seen at baseline. Interaction between GPS and treatment was not significant; however, there was significant interaction between one constituent SNP, FADS1 rs174546 C/T and treatment. After the $1.8 \mathrm{~g} /$ day dose plasma, TG concentration decreased significantly more in $T$-allele carriers than in non-carriers.

We previously found a $15 \%$ reduction in $\mathrm{TG}$ in the female MARINA participants after $1.8 \mathrm{~g} /$ day, showing a clear dose-response relationship with increasing intake $(P=0.002)$, but no significant effects on TC, LDL-C or HDL-C (Sanders et al. 2011). Considerable inter-individual variability in response to n-3 PUFA treatment has been observed (Minihane 2010). This suggests that a range of gene variants may underpin variability in n-3 PUFA metabolism that is influenced by changes in EPA and DHA availability.

We selected SNPs strongly associated with plasma lipids in published GWAS (Kathiresan et al. 2008, 2009; Willer et al. 2008; Aulchenko et al. 2009; Sabatti et al. 2009; Teslovich et al. 2010). In MARINA subjects at baseline, effects of risk alleles were much smaller than those reported in GWAS and not directionally consistent. For example, carriage of the FADS1 SNP rs174546 risk allele appeared to decrease TG at baseline by 0.003 (SE $0.053) \mathrm{mmol} / \mathrm{L}(n=268, P=0.95)$, an anomalous result which underlines the lack of power provided by a single SNP in a small sample. The effect reported in the GWAS was an increase of 0.04 (SE 0.004) $\mathrm{mmol} / \mathrm{L}(n=96,598$, $\left.P=5.41 \times 10^{-24}\right)($ Teslovich et al. 2010). The effects of only two individual SNP alleles, on TC and HDL-C, reached significance in our study. Risk alleles for SNPs discovered in GWAS involving subjects of European ancestry are not necessarily unfavourable in subjects of different ethnicity. As $\sim 20 \%$ of our sample was nonwhite, ethnic heterogeneity could explain directional inconsistencies with GWAS based on subjects of European origin. Ten of our 12 SNPs were among 95 associated with plasma lipids identified by Teslovich et al. (2010) in $\sim 100,000$ Europeans in their primary study. In further analysis of 15,000 East Asians, 9,000 South Asians, 8,000 African Americans and 7,000 additional Europeans, there was no evidence for heterogeneity of effects between the European groups and each of the non-European groups for most of the identified loci. We conclude that inconsistencies between directional effects of risk alleles between GWAS and MARINA subjects are unlikely to originate in ethnic heterogeneity. Regarding response to dietary intervention, in a study sample comprising $80 \%$ whites, $9.5 \%$ South and South East Asian and $8 \%$ black African participants (2.5\% of uncertain ancestry excluded) Walker et al. (2011) investigated GPS association with plasma lipid response to reduced SFA intake in 490 participants. They found no evidence of heterogeneity across the three ethnic groups for the effect of any GPS on the change in plasma lipid traits $(P>0.05)$. We included ethnicity and its interaction with treatment in all our models and found no significant effect.

The difficulty of establishing significant associations with single SNPs has led investigators to turn to panels of SNPs to assess the risk of developing polygenic disease based on quantitative markers (Lamina et al. 2012; Yiannakouris et al. 2012; Isaacs et al. 2013; Rukh et al. 2013; Walker et al. 2013 are recent examples). The GPS is based on the additive effect of risk alleles carried and potentially offers more power than single SNPs as a predictor of dyslipidaemia. The higher the GPS, the less favourable the lipid profile. The predictive utility of GPS reflects both the number and strength of the phenotypic associations of included SNPs and the number of subjects with full genotype data for the GPS determination. We excluded all subjects with more than one missing genotype out of 12, which reduced the sample for analysis from 310 to 252. We found the effects per risk allele were directionally as predicted. The effect for LDL-C-GPS was not significant, approached significance for TC and HDL-C, but in the case of TG was highly significant. In contrast to the insignificant effects of individual TG risk alleles, an additive score based on eight SNPs was highly predictive of plasma concentration at baseline.

We found no significant effects on plasma concentration per GPS risk allele after 12-month treatment with EPA and DHA for any lipid, although the deleterious effects of risk alleles on all were reduced. The greatest impact was seen on TG, for which each additional risk allele was associated with lower plasma concentration after treatment, compared to higher at baseline. However, as interaction between GPS and treatment was not significant, the reduction in plasma TG associated with risk allele carriage cannot be attributed to treatment with EPA and DHA. Studies of similar design by Walker et al. (2011, 2013) have similarly failed to establish predictive power of GPS in the response to dietary interventions to improve plasma lipids. 
As expected, due to small sample size and potential insufficient statistical power, we found no significant effects of single SNP risk alleles on plasma TG before or after treatment. Despite this, we found interaction between FADS1 rs174546 genotype and treatment as a significant determinant of plasma TG concentration. After the $1.8 \mathrm{~g} /$ day dose, plasma TG concentration in non-carriers of the risk allele decreased by $3.5 \%$ from baseline, whereas in carriers, the decrease was $21.6 \%$ and the difference between treatments in this group was highly significant. Fatty acid desaturase 1 encoded by the FADS1 gene is a key enzyme in endogenous desaturation of long-chain fatty acids (Lattka et al. 2010). GWAS have identified several loci in the FADS gene cluster, at which minor alleles were associated with lower TC (Aulchenko et al. 2009; Teslovich et al. 2010), LDL-C (Aulchenko et al. 2009; Sabatti et al. 2009; Chasman et al. 2008; Teslovich et al. 2010), HDL-C (Chasman et al. 2008; Kathiresan et al. 2009) and higher TG concentrations (Kathiresan et al. 2009; Teslovich et al. 2010), suggesting that altered desaturase activities may impact plasma lipid levels. Estimates suggest that minor alleles are associated with lower activity (Zietemann et al. 2010). A number of studies have suggested that dietary intake of different PUFAs interacts with FADS1 variation to affect blood lipids ( $\mathrm{Lu}$ et al. 2010; Cormier et al. 2012; Hellstrand et al. 2012; Standl et al. 2012). We previously found that SNPs rs174537, rs174561 and rs3834458 in the FADS1-FADS2 gene cluster were strongly associated with proportions of a number of plasma n-3 PUFAs at baseline in MARINA subjects (Al-Hilal et al. 2013). EPA and DHA treatment significantly increased estimated desaturase activity after 6 months, and interaction between rs174537 genotype and treatment was a significant determinant. These three SNPs are in strong LD with rs174546, but we found no significant associations with plasma lipids. Two previous studies have investigated interaction between rs174546 and n-3 PUFA intake as a determinant of plasma TG. Standl et al. (2012) found that intake assessed by FFQ did not interact with genotype to alter TG significantly in 2006 children. Cormier et al. (2012) found a significant effect of genotype on plasma TG concentration at baseline $(P=0.02, n=208)$. After supplementation with $3 \mathrm{~g}$ /day EPA and DHA for 6 weeks, a greater decrease observed in risk allele carriers was not significant after adjustment for covariates. The significant gene $x$ treatment interaction and decreased TG in MARINA carriers after adjustment may reflect the longer period of treatment.

The main strength of our study lay in use of controlled doses of EPA and DHA over a 12-month period, with verified intake and long-term compliance. The study was limited by the relatively small sample for post hoc genetic analysis and a suboptimal genotyping success rate. In combination, these factors reduced the power of the study to detect some significant genotype associations and interactions. The observed per allele effect of GPS on change in TG after treatment would be practically impossible to detect with $80 \%$ power. The genotyping success rate at 87-92\% was not as high as expected, but failures were random, as genotype distributions did not deviate from Hardy-Weinberg expectations. Fifty-eight out of 310 participants with more than one of 12 genotypes missing were excluded from the GPS analysis. Twenty-nine of the remaining 252 participants had one missing genotype only, for whom the average count of risk alleles for the respective SNP was substituted. The approach has been used elsewhere (Walker et al. 2011, 2013), but we acknowledge the introduction of this potential inaccuracy in calculating the GPS

The only significant effects at $P<0.05$ were found in relation to one phenotype, TG. Adopting a more stringent approach, a Bonferroni correction to take account of testing eight independent SNPs would lead to a corrected alpha level of 0.0063 . This would remove significance of FADS rs174546 interaction with treatment $(P=0.026)$, and the difference in response to doses seen in carriers would have been marginal $(P=0.007)$. Our results are therefore provisional, and replication would offer the most reliable confirmation. As suggestive evidence accumulates from small studies, prospective recruitment of larger genotype groups will be warranted to explore the effects of common SNPs on the outcome of dietary interventions.

In conclusion, our study does not provide evidence for n-3 PUFA intake in modifying genetic susceptibility to dyslipidaemia measured as GPS. However, this exploratory study suggests that at least one risk allele may interact with diet in determining plasma TG concentration. Increased intake of EPA and DHA was successful in lowering plasma TG, most notably in those genetically predisposed to dyslipidaemia.

Acknowledgments This study was funded by a grant from the Food Standards Agency (United Kingdom) and the Department of Health via the National Institute for Health Research Comprehensive Biomedical Research Centre Award to Guy's \& St Thomas' NHS Foundation Trust in partnership with King's College London (Project Code N02041). The study oils were kindly supplied by Croda Chemicals Europe Ltd. AA was supported by a studentship from the Saudi Arabian Ministry of Higher Education. The assistance of Roy Sherwood and Tracy Dew in the Clinical Biochemistry Department at King's College Hospital and Robert Gray at King's College London is gratefully acknowledged.

Conflict of interest Aseel AlSaleh, Zoitsa Maniou, Fiona Lewis, Wendy Hall, Thomas Sanders and Sandra O'Dell declare that they have no conflict of interest.

Ethical standard All procedures followed were in accordance with the ethical standards of the responsible committee on human 
experimentation (institutional and national) and with the Helsinki Declaration of 1975, as revised in 2000. Informed consent was obtained from all patients for being included in this study.

\section{References}

Al-Hilal M, AlSaleh A, Maniou Z, Lewis FJ, Hall WL, Sanders TA, O'Dell SD, MARINA Study Group (2013) Genetic variation at the FADS1-FADS2 gene locus influences delta-5 desaturase activity and LC-PUFA proportions after fish oil supplement. J Lipid Res 54:542-551

Aulchenko YS, Ripatti S, Lindqvist I, Boomsma D, Heid IM, Pramstaller PP, Penninx BW, Janssens AC, Wilson JF, Spector T et al (2009) Loci influencing lipid levels and coronary heart disease risk in 16 European population cohorts. Nat Genet 41:47-55

Chasman DI, Paré G, Zee RY, Parker AN, Cook NR, Buring JE, Kwiatkowski DJ, Rose LM, Smith JD, Williams PT, Rieder MJ, Rotter JI, Nickerson DA, Krauss RM, Miletich JP, Ridker PM (2008) Genetic loci associated with plasma concentration of lowdensity lipoprotein cholesterol, high-density lipoprotein cholesterol, triglycerides, apolipoprotein A1, and Apolipoprotein B among 6382 white women in genome-wide analysis with replication. Circ Cardiovasc Genet 1:21-30

Corella D, Ordovas JM (2005) Single nucleotide polymorphisms that influence lipid metabolism: interaction with dietary factors. Annu Rev Nutr 25:341-390

Cormier H, Rudkowska I, Paradis AM, Thifault E, Garneau V, Lemieux S, Couture P, Vohl MC (2012) Association between polymorphisms in the fatty acid desaturase gene cluster and the plasma triacylglycerol response to an n-3 PUFA supplementation. Nutrients 4:1026-1041

Delgado-Lista J, Perez-Martinez P, Lopez-Miranda J, Perez-Jimenez F (2012) Long chain omega-3 fatty acids and cardiovascular disease: a systematic review. Br J Nutr 107(Suppl 2):S201-S213

Hellstrand S, Sonestedt E, Ericson U, Gullberg B, Wirfält E, Hedblad B, Orho-Melander M (2012) Intake levels of dietary long-chain PUFAs modify the association between genetic variation in FADS and LDL-C. J Lipid Res 53:1183-1189

International HapMap project http://www.hapmap.ncbi.nlm.nih.gov/. Accessed Dec 2012

Isaacs A, Willems SM, Bos D, Dehghan A, Hofman A, Ikram MA, Uitterlinden AG, Oostra BA, Franco OH, Witteman JC, van Duijn CM (2013) Risk scores of common genetic variants for lipid levels influence atherosclerosis and incident coronary heart disease. Arterioscler Thromb Vasc Biol 33:2233-2239

Janssens AC, Moonesinghe R, Yang Q, Steyerberg EW, van Duijn CM, Khoury MJ (2007) The impact of genotype frequencies on the clinical validity of genomic profiling for predicting common chronic diseases. Genet Med 9:528-535

Jebb SA, Lovegrove JA, Griffin BA, Frost GS, Moore CS, Chatfield MD, Bluck LJ, Williams CM, Sanders TA, RISCK Study Group (2010) Effect of changing the amount and type of fat and carbohydrate on insulin sensitivity and cardiovascular risk: the RISCK (Reading, Imperial, Surrey, Cambridge, and Kings) trial. Am J Clin Nutr 92:748-758

Kathiresan S, Melander O, Guiducci C, Surti A, Burtt NP, Rieder MJ, Cooper GM, Roos C, Voight BF, Havulinna AS, Wahlstrand B, Hedner T, Corella D, Tai ES, Ordovas JM, Berglund G, Vartiainen E, Jousilahti P, Hedblad B, Taskinen MR, NewtonCheh C, Salomaa V, Peltonen L, Groop L, Altshuler DM, OrhoMelander M (2008) Six new loci associated with blood low-density lipoprotein cholesterol, high-density lipoprotein cholesterol or triglycerides in humans. Nat Genet 40:189-197

Kathiresan S, Willer CJ, Peloso GM, Demissie S, Musunuru K, Schadt EE, Kaplan L, Bennett D, Li Y, Tanaka T et al (2009) Common variants at 30 loci contribute to polygenic dyslipidemia. Nat Genet 41:56-65

Kromhout D, Giltay EJ, Geleijnse JM, Alpha Omega Trial Group (2010) n-3 fatty acids and cardiovascular events after myocardial infarction. N Engl J Med 363:2015-2026

Lamina C, Forer L, Schönherr S, Kollerits B, Ried JS, Gieger C, Peters A, Wichmann HE, Kronenberg F (2012) Evaluation of gene-obesity interaction effects on cholesterol levels: a genetic predisposition score on HDL-cholesterol is modified by obesity. Atherosclerosis 225:363-369

Lattka E, Illig T, Koletzko B, Heinrich J (2010) Genetic variants of the FADS1 FADS2 gene cluster as related to essential fatty acid metabolism. Curr Opin Lipidol 21:64-69

Li S, Zhao JH, Luan J, Luben RN, Rodwell SA, Khaw KT, Ong KK, Wareham NJ, Loos RJ (2010) Cumulative effects and predictive value of common obesity-susceptibility variants identified by genome-wide association studies. Am J Clin Nutr 91:184-190

Lu Y, Feskens EJ, Dollé ME, Imholz S, Verschuren WM, Müller M, Boer JM (2010) Dietary n-3 and n-6 polyunsaturated fatty acid intake interacts with FADS1 genetic variation to affect total and HDL-cholesterol concentrations in the Doetinchem Cohort Study. Am J Clin Nutr 92:258-265

Marais AD (2013) Dietary lipid modification for mild and severe dyslipidaemias. Proc Nutr Soc 72:337-341

Minihane AM (2010) Fatty acid-genotype interactions and cardiovascular risk. Prostaglandins Leukot Essent Fatty Acids 82:259-264

Minihane AM (2013) Fish oil omega-3 fatty acids and cardiometabolic health, alone or with statins. Eur J Clin Nutr 67:536-540

Mozaffarian D, Wu JH (2011) Omega-3 fatty acids and cardiovascular disease: effects on risk factors, molecular pathways, and clinical events. J Am Coll Cardiol 58:2047-2067

NCBI SNP database http://www.ncbi.nlm.nih.gov/projects/SNP/ Accessed Dec 2012

ORIGIN Trial Investigators, Bosch J, Gerstein HC, Dagenais GR, Díaz R, Dyal L, Jung H, Maggiono AP, Probstfield J, Ramachandran A, Riddle MC, Rydén LE, Yusuf S (2012) n-3 fatty acids and cardiovascular outcomes in patients with dysglycemia. N Engl J Med 26(367):309-318

Pérusse L, Rice T, Després JP, Bergeron J, Province MA, Gagnon J, Leon AS, Rao DC, Skinner JS, Wilmore JH, Bouchard C (1997) Familial resemblance of plasma lipids, lipoproteins and postheparin lipoprotein and hepatic lipases in the HERITAGE Family Study. Arterioscler Thromb Vasc Biol 17:3263-3269

Rizos EC, Ntzani EE, Bika E, Kostapanos MS, Elisaf MS (2012) Association between omega-3 fatty acid supplementation and risk of major cardiovascular disease events: a systematic review and meta-analysis. JAMA 308:1024-1033

Rukh G, Sonestedt E, Melander O, Hedblad B, Wirfält E, Ericson U, Orho-Melander M (2013) Genetic susceptibility to obesity and diet intakes: association and interaction analyses in the Malmö Diet and Cancer Study. Genes Nutr 8:535-547

Sabatti C, Service SK, Hartikainen AL, Pouta A, Ripatti S, Brodsky J, Jones CG, Zaitlen NA, Varilo T, Kaakinen M, Sovio U, Ruokonen A, Laitinen J, Jakkula E, Coin L, Hoggart C, Collins A, Turunen H, Gabriel S, Elliot P, McCarthy MI, Daly MJ, Järvelin, Freimer NB, Peltonen L (2009) Genome-wide association analysis of metabolic traits in a birth cohort from a founder population. Nat Genet 41:35-46

Sanders TA, Hall WL, Maniou Z, Lewis F, Seed PT, Chowienczyk PJ (2011) Effect of low doses of long-chain n-3 PUFAs on 
endothelial function and arterial stiffness: a randomized controlled trial. Am J Clin Nutr 94:973-980

Standl M, Lattka E, Stach B, Koletzko S, Bauer CP, von Berg A, Berdel D, Krämer U, Schaaf B, Röder S, Herbarth O, Buyken A, Drogies T, Thiery J, Koletzko B, Heinrich J, GINIplus Study Group, LISAplus Study Group (2012) FADS1 FADS2 gene cluster, PUFA intake and blood lipids in children: results from the GINIplus and LISAplus studies. PLoS ONE 7:e37780

Teslovich TM, Musunuru K, Smith AV, Edmondson AC, Stylianou IM, Koseki M, Pirruccello JP, Ripatti S, Chasman DI, Willer CJ et al (2010) Biological, clinical and population relevance of 95 loci for blood lipids. Nature 466:707-713

Walker CG, Loos RJ, Olson AD, Frost GS, Griffin BA, Lovegrove JA, Sanders TA, Jebb SA (2011) Genetic predisposition influences plasma lipids of participants on habitual diet, but not the response to reductions in dietary intake of saturated fatty acids. Atherosclerosis 215:421-427

Walker CG, Holzapfel C, Loos RJ, Mander AP, Klopp N, Illig T, Caterson ID, Hauner H, Jebb SA (2013) Genetic predisposition to an adverse lipid profile limits the improvement in total cholesterol in response to weight loss. Obesity (Silver Spring) 21:2589-2595
Wallace C, Newhouse SJ, Braund P, Zhang F, Tobin M, Falchi M, Ahmadi K, Dobson RJ, Marçano AC, Hajat C, Burton P, Deloukas P, Brown M, Connell JM, Dominiczak A, Lathrop GM, Webster J, Farrall M, Spector T, Samani NJ, Caulfield MJ, Munroe PB (2008) Genome-wide association study identifies genes for biomarkers of cardiovascular disease: serum urate and dyslipidemia. Am J Hum Genet 82:139-149

Willer CJ, Sanna S, Jackson AU, Scuteri A, Bonnycastle LL, Clarke R, Heath SC, Timpson NJ, Najjar SS, Stringham HM et al (2008) Newly identified loci that influence lipid concentrations and risk of coronary artery disease. Nat Genet 40:161-169

Yiannakouris N, Katsoulis M, Dilis V, Parnell LD, Trichopoulos D, Ordovas JM, Trichopoulou A (2012) Genetic predisposition to coronary heart disease and stroke using an additive genetic risk score: a population-based study in Greece. Atherosclerosis 222:175-179

Zietemann V, Kröger J, Enzenbach C, Jansen E, Fritsche A, Weikert C, Boeing H, Schulze MB (2010) Genetic variation of the FADS1 FADS2 gene cluster and n-6 PUFA composition in erythrocyte membranes in the European Prospective Investigation into Cancer and Nutrition-Potsdam study. Br J Nutr 104:1748-1759 